\title{
Structure Design of Disposable Ocean Temperature Depth Sensing Probe Based on F-P Cavity and FBG
}

\author{
Zhaoyue Liu, Lina Zeng, Ke Xu, Qihan Wu, Yichao Shu, Xin Liao, Zaijin Li, Hao Chen, \\ Zhongliang Qiao, Yi Qu, Guojun Liu, Lin Li*
}

Key Laboratory of Laser Technology and Optoelectronic Functional Materials of Hainan Province, College of Physics and Electronic Engineering, Hainan Normal University, Haikou, China

Email: *licust@126.com

How to cite this paper: Liu, Z.Y., Zeng, L.N., Xu, K., Wu, Q.H., Shu, Y.C., Liao, X., Li, Z.J., Chen, H., Qiao, Z.L., Qu, Y., Liu, G.J. and Li, L. (2021) Structure Design of Disposable Ocean Temperature Depth Sensing Probe Based on F-P Cavity and FBG. Journal of Applied Mathematics and Physics, 9, 2947-2953.

https://doi.org/10.4236/jamp.2021.911188

Received: October 28, 2021

Accepted: November 20, 2021

Published: November 23, 2021

\begin{abstract}
The basic principle of sensing is the combination of F-P cavity interference and fiber grating reflection. A hybrid structure sensor probe has been designed based on the combination of an F-P cavity of liquid-filled thermometer structure, and a fiber grating with an elastic diaphragm, herein F-P cavity is used for temperature sensing, and the fiber grating is used for pressure sensing. By adopting the dual optical path structure, the dual-parameter detection of temperature and depth is realized, which solves the problem of low accuracy caused by the cross response of temperature and pressure of a single sensor device and the calculation of the depth information of the ocean with empirical formulas. Compared with traditional sensors, the sensitivity is effectively improved. Theoretical analysis shows that the sensitivity of the F-P cavity with a thermometer structure filled with kerosene reaches 1.334 $\mathrm{nm} /{ }^{\circ} \mathrm{C}$, and the depth sensitivity of the fiber grating is $284.6 \mathrm{pm} / \mathrm{Mpa}$ within the ocean depth range of $0-400 \mathrm{~m}$.
\end{abstract}

\section{Keywords}

F-P Cavity, Fiber Grating, Laser Sensing, Liquid Filling

\section{Introduction}

Temperature and depth are the most basic and important parameters in marine physics. With the continuous development of optical technology, optical sensors have been used in marine resource development, climate monitoring, military industry and other fields [1] [2] [3] [4]. Among them, the fiber grating and F-P cavity structure are the most widely studied objects in optical sensing due to 
their strong anti-interference ability, high sensitivity, small size, intrinsic insulation, etc. [5]. Expendable bathythermograph (XBT) is a commonly used structure in ocean temperature depth sensing, its sensitivity and response time are the most important technical parameters. For traditional sensing probes, which generally use electrical sensing methods, and its response time is on the order of $100 \mathrm{~ms}$, there is a risk of large errors and short circuits because the depth detection is estimated by the falling time of the probe [6] [7].

In 2018, Yang Yu [8] and others prepared an ultra-fine fiber coupler (OMC) using two twisted traditional communication fibers, providing a promising method for improving the temperature sensitivity. Experiments show that the sensor can measure salinity, temperature and depth in seawater under a pressure of 0 - $25 \mathrm{Mpa}$, with temperature sensitivity is $2.326 \mathrm{~nm} /{ }^{\circ} \mathrm{C}$, and pressure sensitivity is $169 \mathrm{pm} / \mathrm{Mpa}$.

In 2019, Yong Zhao et al. [9] used a gold film coated on the surface of an optical fiber to excite the plasmon resonance effect, and two sensitive films (PDMS, SU-8) were plated on the outside of the Au film at different positions. The temperature sensitivity measured by the experiment is $-1.82 \mathrm{~nm} /{ }^{\circ} \mathrm{C}$, and the depth sensitivity is $2.838 \mathrm{~nm} / \mathrm{MPa}$. This structure has great practical significance.

In 2020, Liang Cao [10] and others used ultra-fine fiber couplers combined with fiber optic loop (MCFL) reflective photonic devices for seawater temperature and depth sensing experiments. MCFL's reflective composite sensing optical path design eliminates the effect of MC free port pair on the sensor, and the temperature sensitivity of $248.2 \mathrm{pm} /{ }^{\circ} \mathrm{C}$ and the depth sensitivity of $122.6 \mathrm{pm} / \mathrm{Mpa}$ is obtained. This structure provides a new design for the marine environmental parameter sensor based on the micro-fiber coupler.

In 2021, Hongchang Deng [11] and others proposed a high-sensitivity fiber grating strain and temperature sensor composed of input SMF, HCOF and output SMF cascade structure. Experiments show that the strain sensitivity can reach $-9.29 \mathrm{pm} / \mu$ and then the temperature sensitivity can reach $17.14 \mathrm{pm} /{ }^{\circ} \mathrm{C}$. If this structure is further improved, it will have the potential for high-sensitivity detection of ocean depths.

The above research shows that the use of optical fibers with different structures and special processes for coupling and coating will greatly improve the sensitivity of the sensor device, but the response time of sensing is seldom addressed, and the complex process will reduce the stability and repetition of the sensor device. The temperature and pressure in the same device will be sensitive to cross transmission, which will affect the overall sensing accuracy.

This paper proposes an ocean temperature depth sensing structure for XBT application. The structure is based on the parallel connection of FP cavity and Fiber-Brag-Grating (FBG). The temperature and depth are measured by the FP cavity part of the thermometer structure and the FBG incorporated with the elastic diaphragm. Theoretical analysis and calculation, a sensor structure with good temperature sensitivity and depth sensitivity have been designed, which provides a feasible solution for further application on XBT. 


\section{Sensor Probe Design}

The F-P cavity and the FBG are connected in parallel, and the specific optical path structure of the sensing system is shown in Figure 1.

The part of the FP cavity, used to measure temperature, is first filled with liquid into the microcapillary. One end of the thin tube is inserted with SMF and the port is welded, and the other end is welded with a cylindrical quartz tube filled with water to form a seal. The liquid surface and the end face of the SMF constitute the FP cavity structure. In the FBG part used to measure pressure, the FBG with one end connected to the spring is connected to the elastic diaphragm, as indicated. Since the outside will be encapsulated by a steel cylinder, the lateral stress is largely overcome, so this article ignores the influence of the lateral stress, the probe structure diagram as shown in Figure 2.

In Figure 2(a), the thermometer-like structure can amplify the influence of temperature on the length of the cavity through the thermal expansion effect of the liquid. The cylindrical quartz tube at the back end is in contact with the outside world, and the large contact area can speed up the heat exchange with the outside world, reducing the response time of sensing. In Figure 2(b), the elastic diaphragm is in contact with seawater, and the stress on the FBG becomes axial. A large contact area can increase the stress effect of seawater on the FBG, and enhance the sensitivity of the depth sensing of the structure.

\section{Theoretical Analysis and Calculation}

\subsection{Theoretical Analysis of Temperature Sensing Structure}

In the fiber optic FP temperature sensing structure, two reflective surfaces (the reflectivity of which are $R_{1}$ and $R_{2}$ respectively) form an F-P cavity with a length of $L$. If $R_{1}=R_{2}=R$ and the refractive index of the F-P cavity is $n$, when the intensity and When a light beam with wavelengths $\lambda$, intensity $I_{0}$, enters the F-P cavity, the reflected light intensity $I_{R}$ is

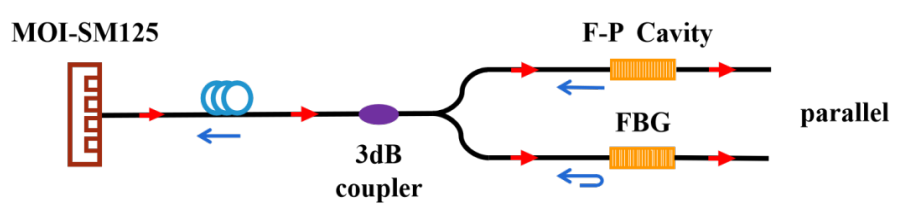

Figure 1. F-P cavity and FBG structure light path diagram.

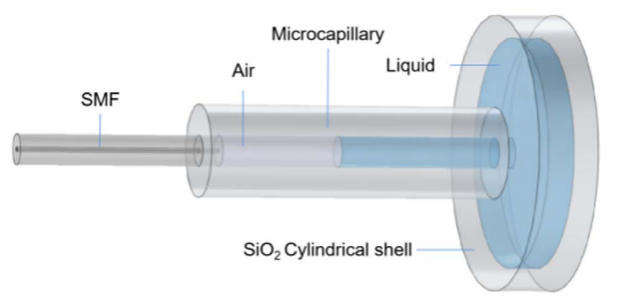

(a)

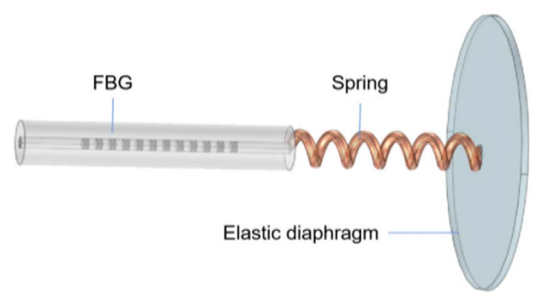

(b)

Figure 2. Schematic diagram of sensing structure. (a) F-P cavity; (b) FBG. 


$$
I_{R}=\frac{2 R[1-\cos (4 \pi n L / \lambda)]}{1+R^{2}-2 R \cos (4 \pi n L / \lambda)} \cdot I_{0}
$$

When the reflectivity $R$ of the reflecting surface is small, the multi-beam interference degenerates into two-beam interference, and the reflected light intensity can be simplified as

$$
I_{R}=2 R[1-\cos (4 \pi n L / \lambda)] \cdot I_{0}
$$

$m$ is a positive integer, indicating the ordinal number of the interference fringe trough, the cavity length of the F-P cavity can be obtained by the center wavelength of two adjacent troughs

$$
L=\frac{\lambda_{\min 1} \lambda_{\min 2}}{2 n\left(\lambda_{\min 1}-\lambda_{\min 2}\right)}
$$

The center wavelength $\lambda_{\min }$ of the interference fringe trough satisfies

$$
2 m \pi=4 \pi n L / \lambda_{\min }
$$

The filling liquid selected here is pure water, glycerol and kerosene. The thermal expansion coefficient changes little in the temperature range of $10^{\circ} \mathrm{C}-30^{\circ} \mathrm{C}$, and the refractive index of the liquid surface is similar to that of the fiber core. It is easy to fill at room temperature with low cost. The basic parameters of the three liquids are shown in Table 1.

The center wavelength of the selected light source is $1550 \mathrm{~nm}$. Considering that the temperature range of underwater $0-400 \mathrm{~m}$ sensing is generally between $10^{\circ} \mathrm{C}-30^{\circ} \mathrm{C}$, we here set the temperature range of the sensing structure as $5^{\circ} \mathrm{C}$ $35^{\circ} \mathrm{C}$. The microcapillary's specifications are $128 \mu \mathrm{m}$ ID, $420 \mu \mathrm{m}$ OD, the cladding diameter of the single-mode fiber is $125 \mu \mathrm{m}$, the inner radius and height of the rear quartz cylindrical shell are $650 \mu \mathrm{m}$ and $130 \mu \mathrm{m}$, respectively. $2 \mathrm{~mm}$ long microcapillary is intercepted as calculated according to the specifications of the selected material. The appropriate volume of the filled liquid is $1.8 \times 10^{-1} \mathrm{~mm}^{3}$, (here the main consideration is that the cavity length is too short, which will cause the pressure of the air column in the cavity to be large, so as to affect the linear change of the interference cavity length). During the expansion of the liquid, the equation of the change of the cavity length $\mathrm{L}$ after $1^{\circ} \mathrm{C}$ can be expressed as

$$
\Delta L=2 \Delta V / 15 \pi(I D)^{2}
$$

Then the change of wavelength with every $1^{\circ} \mathrm{C}$ change is

$$
\Delta \lambda=\frac{15 \pi(I D)^{2}\left(\lambda_{\min 1} \lambda_{\min 2}-\lambda_{\min 2} \lambda_{\min 3}\right)}{4 n \Delta}
$$

Table 1. Thermal expansion coefficients of three sensitizing liquids.

\begin{tabular}{cccc}
\hline Liquid & water & Glycerine & Kerosene \\
\hline Coefficient of thermal expansion $(1 / \mathrm{K})$ & 0.000208 & 0.0050 & 0.0010 \\
\hline
\end{tabular}


According to the sensing range, the overall wavelength drift is deduced, then according to the above formula, the temperature-wavelength relationship for the three different sensitizing liquids can be obtained as shown in Figure 3.

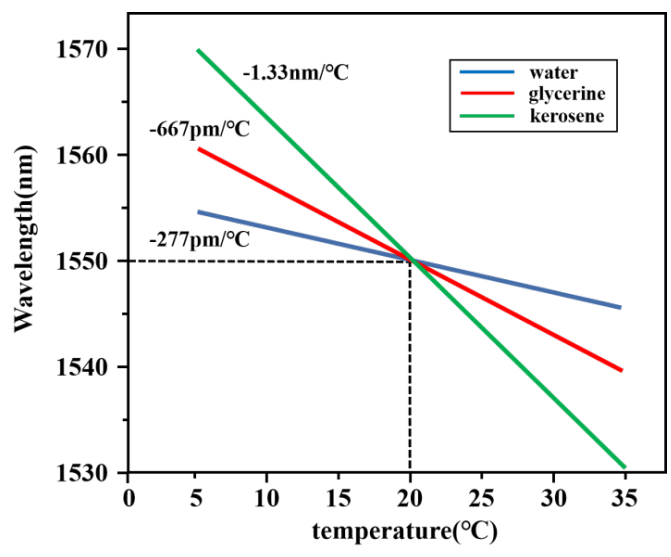

Figure 3. The relationship between temperature and wavelength in the sensing structure.

After calculation, it can be obtained that the temperature sensitivity of the F-P cavity is $277.4 \mathrm{pm} /{ }^{\circ} \mathrm{C}, 666.8 \mathrm{pm} /{ }^{\circ} \mathrm{C}$, and $1.334 \mathrm{~nm} /{ }^{\circ} \mathrm{C}$ for pure water, glycerol and kerosene as the sensitizing liquids, respectively.

\subsection{Theoretical Analysis of Depth Sensing Structure}

In the FBG part used for pressure sensing, the central reflection wavelength of the fiber Bragg grating depends on the period of the fiber grating and the effective refractive index of the fiber core. The equation can be expressed as

$$
\lambda=2 n_{\text {eff }} \Lambda
$$

The change of external stress will cause the center wavelength of the fiber Bragg grating to shift, and its expression is

$$
\Delta \lambda_{b}=2\left(\Delta n_{\text {eff }} \Lambda+n_{\text {eff }} \Delta \Lambda\right)
$$

In the formula, $n_{\text {eff }}$ represents the effective refractive index. When the external uniform axial stress acts on the fiber grating, it will cause the axial strain to change the effective refractive index of the fiber core. At the same time, the axial strain will also change the grating period $\Lambda$, the expression is

$$
\epsilon=\frac{\Delta \Lambda}{\Lambda}
$$

Then the wavelength change caused by the axial strain can be expressed as

$$
\Delta \lambda_{b}=2\left(1-P_{\varepsilon}\right) \lambda \varepsilon
$$

$P_{\varepsilon}$ in the equation is the effective elasticity coefficient. The parameters of the fiber grating to be used are: the central reflection wavelength is $1550 \mathrm{~nm}$, the core diameter is $8.2 \mu \mathrm{m}$, the cladding diameter is $125 \mu \mathrm{m}$, the grating length is 10 $\mathrm{mm}$, and the grating tensile limit is $3000 \mu \varepsilon . P_{\varepsilon}=0.22$. So its strain sensitivity is 
$1.2 \mathrm{pm} / \mu \varepsilon$.

We choose to use a stainless steel elastic diaphragm with a thickness of $h=$ $0.45 \mathrm{~mm}$ and a radius of $R=5 \mathrm{~mm}$. The effective length of the fiber grating is 3 $\mathrm{mm}$. For the ocean pressure range of $0.1-5 \mathrm{MPa}$, the pressure sensitivity of the FBG to be designed is calculated as $284.6 \mathrm{pm} / \mathrm{Mpa}$.

\section{Conclusion}

In this paper, an expendable ocean temperature probe structure based on F-P cavity and FBG is designed. According to the application requirements of XBT fast sensing, the theoretical analysis and simulation of temperature sensitivity, depth sensitivity and response time are carried out. The sensitivity effects of several different sensitizing liquids and the addition of elastic diaphragms on the sensing structure were compared, and the temperature sensitivity of 277.4 $\mathrm{pm} /{ }^{\circ} \mathrm{C}, 666.8 \mathrm{pm} /{ }^{\circ} \mathrm{C}, 1.334 \mathrm{~nm} /{ }^{\circ} \mathrm{C}$, and the depth sensitivity of $284.6 \mathrm{pm} / \mathrm{Mpa}$ were obtained for pure water, glycerol and kerosene as the sensitizing liquids, respectively. It testified the theoretical feasibility of using this structure to obtain good sensitivity and response time. Under the condition of linear expansion of the liquid in the cavity, the use of a liquid with a high expansion coefficient can enhance the sensitivity of the sensing structure; therefore it is suitable for marine expendable depth and temperature sensor.

\section{Acknowledgements}

This work is supported by Finance science and technology project of Hainan province (ZDYF2020217, ZDYF2020020, ZDYF2020036), Scientific Research Projects of Higher Education Institutions in Hainan Province (Hnjg2021ZD-22, Hnky2020ZD-12, Hnky2020-24), Hainan Provincial Natural Science Foundation of China (2019RC190, 2019RC192, 120MS031), Major Science and Technology Project of Hainan Province (ZDKJ2019005), National Natural Science Foundation of China $(62174046,62064004,61964007,61864002)$, Open Fund for Innovation and Entrepreneurship of college students (202111658021X, 202111658022X, 202111658023X, 202111658013) and specific research fund of The Innovation Platform for Academicians of Hainan Province (YSPTZX202127).

\section{Conflicts of Interest}

The authors declare no conflicts of interest regarding the publication of this paper.

\section{References}

[1] Kaczmarek, C. (2019) Photonic Crystal Fiber Sensor for Impulsive Pressure Wave Measurements. Optics and Lasers in Engineering, 122, 23-28. https://doi.org/10.1016/j.optlaseng.2019.05.017

[2] Bringas, F. and Goni, G. (2015) Early Dynamics of Deep Blue XBT Probes. Journal of Atmospheric and Oceanic Technology, 32, 2253-2263.

https://doi.org/10.1175/JTECH-D-15-0048.1 
[3] Abraham, J.P., Cowley, R. and Cheng, L. (2016) Quantification of the Effect of Water Temperature on the Fall Rate of Expendable Bathythermographs. Journal of Atmospheric and Oceanic Technology, 33, 1271-1284. https://doi.org/10.1175/JTECH-D-15-0216.1

[4] Min, R., Liu, Z., Pereira, L., et al. (2021) Optical Fiber Sensing for Marine Environment and Marine Structural Health Monitoring: A Review. Optics \& Laser Technology, 140, Article No. 107082. https://doi.org/10.1016/j.optlastec.2021.107082

[5] Kersey, A.D., Davis, M.A., Patrick, H.J., et al. (1997) Fiber Grating Sensors. Journal of Lightwave Technology, 15, 1442-1463. https://doi.org/10.1109/50.618377

[6] Goes, M., Goni, G. and Dong, S. (2015) An Optimal XBT-Based Monitoring System for the South Atlantic Meridional Overturning Circulation at $34^{\circ} \mathrm{S}$. Journal of Geophysical Research: Oceans, 120, 161-181. https://doi.org/10.1002/2014JC010202

[7] Stark, J., Gorman, J., Hennessey, M., et al. (2011) A Computational Method for Determining XBT Depths. Ocean science, 7, 733-743. https://doi.org/10.5194/os-7-733-2011

[8] Yu, Y., Bian, Q., Lu, Y., et al. (2018) High Sensitivity All Optical Fiber Conductivity-Temperature-Depth (CTD) Sensing Based on an Optical Microfiber Coupler (OMC). Journal of Lightwave Technology, 37, 2739-2747. https://doi.org/10.1109/JLT.2018.2878475

[9] Zhao, Y., Wu, Q. and Zhang, Y. (2019) Simultaneous Measurement of Salinity, Temperature and Pressure in Seawater Using Optical Fiber SPR Sensor. Measurement, 148, Article No. 106792. https://doi.org/10.1016/j.measurement.2019.07.020

[10] Cao, L., Yu, Y., et al. (2020) High Sensitivity Conductivity-Temperature-Depth Sensing Based on an Optical Microfiber Coupler Combined Fiber Loop. Chinese Optics Letters, 18, Article No. 011202. https://doi.org/10.3788/COL202018.011202

[11] Deng, H., Wang, R., Jiang, X., et al. (2021) A Long Period Grating Sensor Based on Helical Capillary Optical Fiber. Journal of Lightwave Technology, 39, 4884-4891. https://doi.org/10.1109/JLT.2021.3075962 